\title{
Ueber Irrenanstalten im Orient und in Australien.
}

Von

\author{
Dr. S. LILIENSTEIN, \\ Arzt fiir innere und Nerven-Kranklleiten in Bad Nauheim (im Winter in Kairo).
}

Wenn ich auf meinen Reisen, die mich rund um die Erde führten, die Gelegenheit wahrnahm, auch für unser Fach, die Neurologie und Psychiatrie, neue Eindrücke zu sammeln und meine Erfahrungen zu bereichern, so bildete ich mir nicht ein, dass ich dabei ernster, wissenschaftlicher Arbeit obliege und mich etwa auf dem Gebiet der ,,vergleichenden Psychiatrie" betätige, aber ich gewann doch die Ueberzeugung, dass durch die persönliche Kenntnisnahme von Einrichtungen und spezicll der Irrenanstalten fremder Länder eine Fülle von Anregung gewonnen werden kamn.

Man sollte annehmen, dass von den Instituten, über die ich hier berichten will, viele sich im Prinzip und wesentlich von unseren deutschen und europäischen Irrenanstalten unterscheiden. Das ist nicht der Fall. Wo immer ich einer fortgeschritteneren Irrenfürsorge begegnete, war sie die Folge der Einbürgerung oder \%um mindesten des starken Einflusses europäischer Kultur. Bei Australien, das von England aus seine Kultur empfing und sie dann allerdings ziemlich unabhängig weitergestaltete, versteht sich das von selbst. Aber auch im Orient entwickelte sich das Irrenwesen fortschrittlich nur in jenen Ländern, wo europäische Staaten eine führende Rolle spielen, wie z. B. in Aegypten, oder wo der Bildungsdrang der Eingeborenen die fremdländische Kultur sich zur Hebung der einheimischen zunutze machte, wie z. B. in Japan.

Wenn einerseits die Anlage der Krankenhäuser im Prinzip fast immer an für uns Bekanntes erinnert und nur jeweils dem Klima und den Verhältnissen des Landes angepasst ist, so stösst man andererseits bei den Wanderungen durch dieselben doch 
gelegentlich auf neue Krankheitsformen. Meist freilich sieht man vielmehr die typischen Psychosen, deren Zustandsbilder uns von unseren Irrenanstalten her bekannt sind, modifiziert durch Eigenart und Lebensgewohnheiten der verschiedenen Völker.

Bei meinem wiederholten Aufenthalt in Aegypten hatte ich Gelegenheit, die Kairiner Irrenanstalt, die einzige des Landes, genauer kennen zu lernen. Sie steht unter englischer Leitung und ist infolgedessen ziemlich modern eingerichtet. Die kahlen Höfe, in denen die Kranken zum Teil beschäftigungslos herumlungern - für uns im allgemeinen das Zeichen einer schlechten Anstaltsleitung - entsprechen dort der Eigenart des Landes, das heisst dem schwer zu bepflanzenden Wüstensandboden und der Trägheit der Bewohner. Der Orientale kommt nicht aus seiner Gewohnheit, wenn er nichts tut. Soweit die Kranken Lust und Fähigkeit zur Arbeit zeigen, finden sie auch in der Irrenanstalt zu Kairo entsprechende Beschäitigung durch leichte Flechtarbeiten etc.

Infolge der unzulänglichen Mittel ist es dem leitenden Art, Dr. Warnock, nicht möglich, für erregte Kranke genügend Aufsichtspersonal anzustellen, und er hält die abgeschlossenen Einzelzellen für unvermeidlich. Als ich mit einem bekannten deutschen Psychiater wieder vor einiger Zeit diese Zellenabteilung besichtigte, äusserte der letztere Dr. Warnock gegenüber sein Missfallen an diesem ,,Restraint". Auf die Frage, was man denn in Deutschland mit sehr erregten Kranken anfange, wiesen wir u. a. auf die Dauerbäder und die verschiedenen narkotischen Mittel hin. Dr. Warnock seinerseits hielt uns die Gefahr der ,,hydrotherapeutischen" und der ,,chemischen Zwangsjacke" entgegen, die ja in der Tat die Scylla gegenüber der Charybdis der Zellenbehandlung bilden.

Interessant ist in der Kairiner Irrenanstalt, wie in Kairo überhaupt, das Völkergemisch, dem man begegnet. Alle Rassen und Nationen sind unter den Kranken vertreten. Nan trifft Fellachen, Nubier, Berber, überhaupt alle Arten von Afrikanern und daneben Asiaten und Europäer jeder Nationalität.

Dass im Bereich des Islam der Allioholismus sehr viel seltener die Ursache von Geisteskrankheiten bildet als bei uns, ist selbstverständlich. Sehr streng wird aber das Abstinenzgebot Mohammeds, besonders in den Städten, nicht mehr gehalten, und unter den 573 Aufnahmen der Kairiner Irrenanstalt im Jahre 1908 befinden sich doch $3 \check{5}$ Alkoholisten. Allerdings konnte ich nicht feststellen, wieviel Nichtmohammedaner darunter waren. Zwei andere Ursachen von Psychosen, die bei uns vollständig fehlen, treten dagegen in der Statistik hervor, nänlich: die Pellagra mit 88, der Haschisch mit 36 von 573 Fällen. Die Pellagra ist ein sehr vielgestaltiges, Cerebral- und Spinalsymptome bietendes Krankheitsbild. Sie tritt meist in armen, ländlichen Distrikten, in denen verdorbener Mais das Hauptnahrungsmittel bildet, auf und führt neben fortschreitender Demenz vor allem auch zu einem raschen körperlichen Verfall, infolge von häufiger, langanhaltender Diarrhöe. 
Ein eigenartiges Symptom der Krankheit ist das Rauhwerden der Haut, die sich dann abhebt und pigmentlose Stellen zurücklïsst. Bei der Behandlung wird jetzt häufig Atoxyl in Anwendung gebracht und nach den Angaben Warnocks Erfolg damit er\%ielt. Dic Pellingra tritt auch anderiärts im Orient und neverdings in den Vereinigten Staaten (Süid-Kilrolina) auf. Die Diagnose bei chronischen Fällen wird durch die ausgedehnten Pigmentatrophien der Haut sehr erleichtert.

Von Aegypten abgesehen, sind die Erkrankungen infolge von Haschisch- und Opiumgenuss im Orient mehr verbreitet als die Pellagra. Die Opium- und Haschischerkiankung entspricht im wesentlichen dem Alkoholismus bei uns; sie ist die Prolctaricrkrankheit der östlichen Hemisphäre. Das Krankheitsbild deckt sich in fast allen Teilen mit dem der 'Trunksucht, angefangen vom akuten Rausch, der zu Lärmszenen in den Opiumspelunken führt, bis zu dem Bild des verkommenen, chronischen Opiumrauchers.

Je weiter man in den Orient eindringt, desto schwieriger lassen sich Einblicke in die Krankheitsverhältnisse überhaupt und die psychiatrischen im besonderen gewinnen. So hat z. B. das grosse chinesische Reich keine einzige öffentliche Irrenanstalt. Die Geisteskianken werden alle in der Familie ,bchandelt". Das führt häufig zu Misshandlungen und elender Vernachlässigung, um so mehr, als der vielfach herrschende Dämonenglinuben sich den Geisteskranken gegenüber geltend macht.

In Japan war mir' Gelegenheit geboten, mich über die Ir'enfürsorge genauer zu informieren ${ }^{1}$. Die Zahl dej Irrenanstalten ist dort noch gering, und diese sind ausnahmslos neneren Datums. Das Land besitzt nur eine einzige staratliche Irrenklinik, das Sugamohospital in 'Tolio. Eine weitere sollte dieses Jahr in Kyoto errichtet werden. Die Leiter der beiden Anstalten, S. Kure und Imamura, haben ihre Ausbildung in Deutschland erhalten, und auch auf weiteren Gebieten der Nedizin macht sich der deutsche Einfluss bemerkbar. In der Hauptsache werden zurzeit die japanischen Geisteskianken noch in Privatanstalten untergebracht, die aber, soweit ich sie kiennen lernte, durchaus modernen Ansprüchen genïgen, da sie ja auch meist erst. neuerdings errichtet wurden.

Der Beginn, der Verlauf und die Prognose der Geisteskrankheiten ist bei den Japanern im allgemeinen ebenso wie bei uns. Die Paralyse ist in Japan relativ selten, so dass im Bestand der Anstalten die endogenen Psychosen, Paranoia, Dementia pratecox, Manie und senile Psychosen mehr in den Vordergrund treten.

Eingehender noch als in Japan konnte ich mich in Australien mit der Irrenfürsorge bekannt machen. Ich besuchte dort alle grösseren staatlichen Irrenanstalten. Allerdings gibt es der'en nur 5 und die einzelnen Aufnahmebezirke umfassen infolgeciessen

$\left.{ }^{1}\right)$ Ich habe darüber bereits in einem Vortrag berichtet, den ich vor der psychiatrischen Gesellschaft in Tokio hielt (publiziert in der Fustschrift für Flechsig, diose Monatsschrift Band XXV.L. Seito 226-232). 
ungeheure Gebiete. Zuweilen müssen die Kranken eine zwei- bis dreiwöchige Schiffsreise zurücklegen, um zur Anstalt zu gelangen, und wonn es sich nicht um sehr dauerhafte, langanhaltende Psychosen handelt, ist es nicht ausgeschlossen, dass die Patienten gesund sind, ehe sie dorthin kommen.

Die Zahl der männlichen Geisteskranken Westaustraliens betrug scit 10 Jahren ungefähr 2-3\% der Bevölkerung, entspricht also dem Prozentsatz bei uns. Früher war er viel geringer, und die weiblichen Anstaltsliranken bilden auch heute noch nur $1 \frac{1}{2} \%$ der weiblichen Bevölkerung. Diese Verhältnisse sind auf die Art der Einwanderung zurückzuführen, die meist aus gesunden, jungen Männern, Irländern, Engländern und Italiener'n besteht, beziw. bis vor kurzem bestanden hat.

In jeder der australischen Irrenanstalten traf ich auch einen oder mehrere Deutsche, ein Beweis dafür, dass auch das deutsche Element, wenn auch nur schwach, in der Bevölkerung vertreten ist.

Die westaustralische Irreninstalt Claremont ist sehr übersichtlich angelegt und modern eingerichtet. Zur Zeit meines Aufenthaltes wurden ca. 300 Patienten dort verpflegt, doch bestand die Absicht, alle Kranken der früheren Anstalt zu Fremantle dorthin zu überführen. Die Anstalt in Fremantle ist nämlich ein etwa 40 Jahre altes Gebäude, das früher als Gefängnis gedient hat, als England noch seine Deportierten nach Australien vorschiclite. Sie entspricht demzufolge nicht mehr den modernen Anforderungen. Die derzeitige Bevöllierung Westaustraliens besteht zu einem grossen Teil aus Nachkommen jener Deportierten, und es ist deshalb nicht verwunderlich, dass sich unter ihr relativ viele Degenerierte beiinden.

Im Parkside Asylum bei Adelaide finden sämtliche Kranke des Starates Südaustralien Unterkommen. Die Anstalt verpflegt zur\%eit über 1000 Kranke und verzeichnete im Jahre 1907211 Neuaufnahmen, die sich aus 131 Australiern, 43 Engländern, 12 Deutschen, () Irländern, \& Schotten und vereinzelten Vertretern anderer Nationalitäten zusammensetzten. Die Anstalt, die ausserhalb der Stadt liegt, ist von. einem prächtigen Park eingeschlossen und besitzt völlig moderne Eimichtungen, obgleich sie schon 1860 erbaut wurde. In ihrem Plan, ihrer Anlage und dem äusseren Eindruck erinnert sie lebhaft an die Frankfurter städtische Irrenanstalt. Die Verpflegung der Kranken ist ausgezeichnet, und Iïr zweckmässige Beschäftigung ist ebenfalls gesorgt. Nur auf der Abteilung für verbrecherische Geisteskranke sah ich das bckannte Bild öder Höfe mit herumhockenden, schlecht gepflegten Kranken.

Die beiden Anstalten des Staates Victoria, Kew und Yarra Bent, befinden sich in der Nähe der Hauptstadt Melbourne. Sie haben zusammen etwa 200 Aufnahmen pro Jahr und einen Bestand von 1600 Kranken. In Keiw wurden nach Angabe des leitenden Arztes, Dr. W. H. Barker, mit Rücksicht auf das subtropische Klima Leinwandzelte als Baricken errichtet, die durch Berieselung 
mit kaltem Wasser ständig liühl erhalten werden liönnen und in der heissen Jahreszeit den Kranlien einen erträglichen Aufenthalt bieten. In einem Zelt, das etwa 2000 Mark kostet, haben ungefähr 15 Kranke Raum.

Die staatlichen und die Privatanstalten, die ich in Sidney, der Hauptstadt des Staates New South Walcs, besichtigte, stehen hinter den oben beschriebenen nicht zurück. Fast überall in Australien hat man seine Freude an den modernen Einrichtungen und dem fortschrittlichen Geist der Anstaltsleiter, die allen Neuerungen und Verbesserungen ihr Interesse zuwenden. Anstalten, die schon vor 30 bis 40 Jahren errichtet wurden, wie das (allian Park Asylum in Sidney (mit etwa 1000 Kranken), werden ständig reorganisiert. Um diese Anstalt speziell hat sich Dr. Chisholm Ross grosse Verdienste erworben. In mustergïltiger Weise werden hier die Kranken so viel als möglich zur Arbeit herangezogen und die Frauen mit Nähen, Hand- und Küchenarbeiten, dic IIänner mit Schusterei und Schneiderei beschä̈tigt.

Ein besonderer Vorzug des Callan Park -Asylum, den ich sonst nirgends fand, besteht darin, dass die Kontrolle über die Kranken nach der Entlassung von den Anstaltsärzten sehr gewissenhaft und erfolgreich ireitergeführt wird. Sie haben zu diesem Zireck cin eigenes System herausgebildet, nach welchem die Angehörigen bei Uebernahme der Kranken einen Revers unterschreiben müssen, in dem sie sich rerpflichten, für ihre Pflegebefohlenen entsprechend zu sorgen und sie regelmässig zur Untersuchung nach der Anstalt zu bringen. Die Besuche werden nach einer Liste kontrolliert. Ein Verstoss gegen die übernommene Verpflichtung richt eine Konventionalstrafe für die Angehörigen, eventucll die Wiederauinahme in die Anstalt nach sich.

Weniger befriedigend als der Besuch der australischen Irrenanstalten verlief derjenige von Ceylon und später derjenige von Singapore. In Colombo scheint die psychiatrische Klinik bedauerlicherweise noch mit dem - Gefängnis eng verbunden zu sein, woraus natürlich gefängnistechnische Gesichtspunkte für die Pflege der Geisteskianken resultieren müssen.

Auch in Singapore wurde man eher an eine Zwangs- als an eine Heilanstalt erimnert. Zwar sind die Kranken der dortigen Anstalt in reinlichen, hellen und luftigen Räumen, für unsere Begriffe aber recht inhuman hinter eisernen Gittern, in kahlen Loggien mit Steinfussböden und auf harten Pritschen untergebracht. Allerdings bedingen die dort herrschenden Temperaturverhältnisse andere Vorkehrungen, als sie bei uns gebräuchlich sind. Singapore ist bekanntlich nur 2 Brcitegrade vom Aequator entfernt; ständig herrscht dort eine feuchtwarme Luft, so dass Hcizvorrichtungen, ïberflüssig und Fenster und luftabschliessende Türen durchinus nicht angebracht wären. Die Temperatur war in ciesen hallenartigen Gebäuden trotz des Steinfussbodens und der Steinwände bei meinem Besuch, Ende Dezember, drückend schwül. In dieser tropischen Luittfeuchtigkeit, die ungefähr nach jecler Viertelstunde 
zu Regenschauern führte, machten sich $25^{0}$ im Schatten schon unangenehm heiss fühlbar., während die gleiche Temperatur in der trockenen Luft Aegyptens äusserst angenehm empfunden wird.

Eine eigentïmliche Art psychischer Erkrankung, die sonst nirgends beobachtet zu werden scheint, lernte ich in Singapore, zwar nicht in der Irrenanstalt, sondern im allgemeinen Krankenhaus kennen. Es handelt sich um eine epidemische Kramptform, die mit retrograder Amnesie einhergeht und meist frauen im mittleren Alter befällt. Die Fälle sollen aus bestimmten Distriliten stammen, Hysterie und psychische Infeltion soll indessen völlig ausgeschlossen sein. Die einzelnen Anfälle gehen mit Bewusstlosigkeit einher und dauern 1-3 Tage.

Wie schon eingangs erwähnt, hatte ich leider keine Zeit, den mich besonders interessierenden Erscheinungen eingehend nachzuforschen, und ich musste mich damit begnügen, mich über das einigermassen zu informieren, was mir im Vorbeigehen ins Auge fiel. Bei dem Blick über meine gesamten Reiseerfahrungen kann ich mich aber dem Eindruck nicht verschliessen, dem ich bereits früher Ausdruck gegeben habe ${ }^{1}$ ), dass die Organisation der Irrenfürsorge am besten in den europäischen Kulturstaaten, und unter diesen wieder hervorragend human in Deutschland durchgeführt ist.

Wir dürfen also auf diese Seite unserer westeuropäischen und speziell deutschen Kultur stolz sein, wenn auch in den ehrwürdigen, alten Kulturen des Orients, in Aegypten und Indien, wahrscheinlich die Wurzeln der unsrigen zu suchen sind. 